\section{Intracardiac extension of lung cancer via the pulmonary vein}

A 69-year-old male heavy smoker had intermittent haemoptysis for 1 month. He did not have exertional dyspnoea, palpitations or chest pain. Chest radiography showed a mass over the right lower lung (RLL). A CT scan of the chest revealed a dumbbell-shaped tumour with an irregular mass in the RLL field, with a tubular part extending along the right inferior pulmonary vein (fig 1A, arrowheads) and an oval tumour in the left atrium (fig 1A, asterisk). Transthoracic echocardiography through the apical four-chamber view showed an intracardiac tumour (asterisk, fig 1B) protruding from the right pulmonary vein (arrowheads, fig 1B). A complete staging investigation, including bronchoscopy, head CT scan and whole body bone scintigraphy, did not show any distant metastasis.

Despite failure to obtain tissue via transthoracic needle biopsy, RLL lobectomy and excision of the left intra-atrial tumour were performed based on the potential life-threatening situation. The surgical specimen (fig 2A) showed the same features as those seen on the chest CT scan. Pathological examination showed small cell lung cancer with cancerous thrombus formation in the pulmonary vein (fig $2 \mathrm{~B}$ ) and left atrium without wall invasion. The patient underwent surgery uneventfully and received adjuvant chemotherapy thereafter.
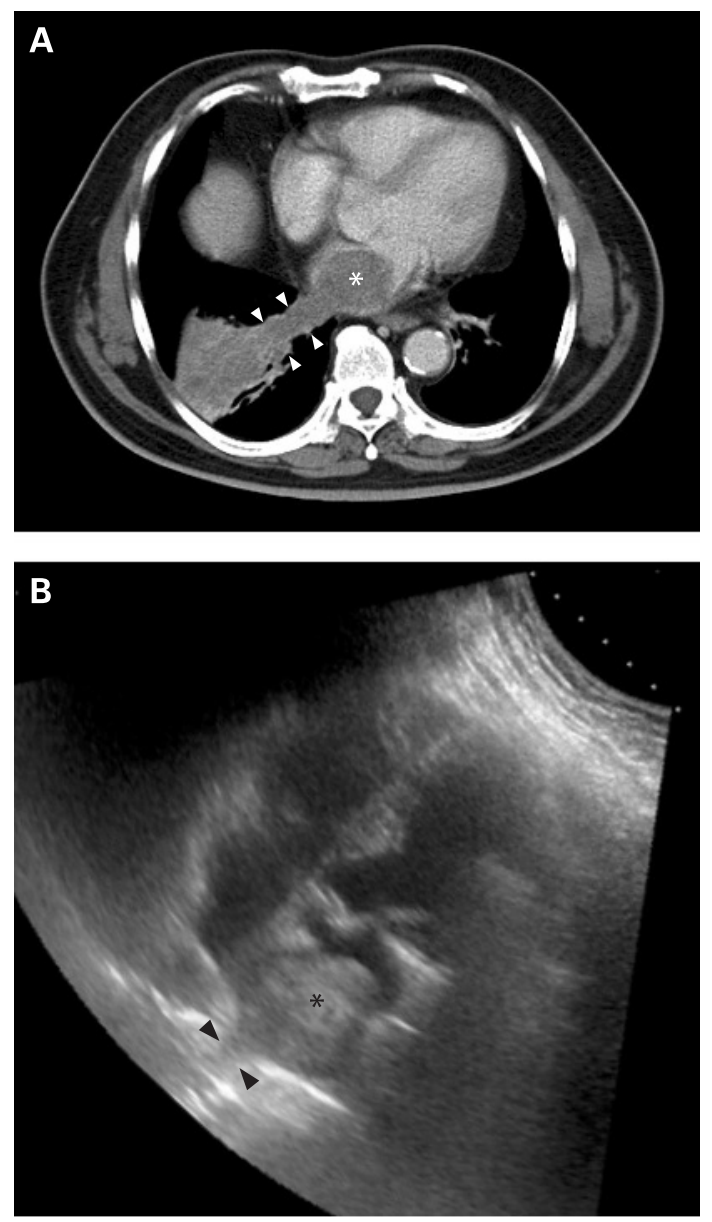

Figure 1 (A) Chest CT scan showing a right lower lung tumour extending through the right inferior pulmonary vein (arrowheads) into the left atrium as an intracardiac tumour (asterisk). (B) Transthoracic echocardiography showing an intracardiac tumour (asterisk) with a short stalk along the pulmonary vein (arrowheads).
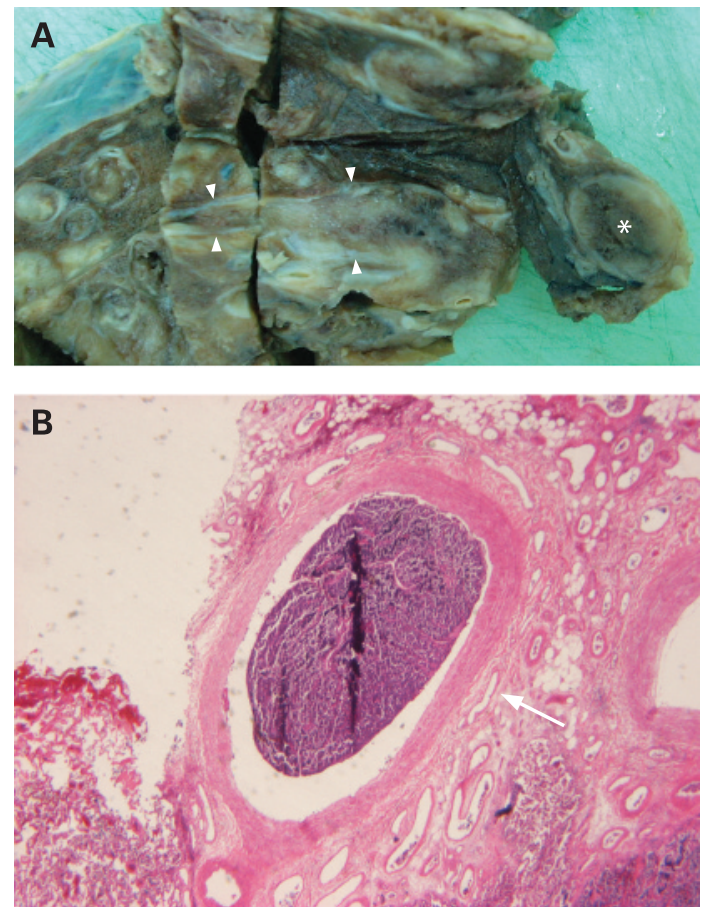

Figure 2 (A) Surgical specimen of right lower lung lobectomy showing cancerous thrombus formation in the right pulmonary vein (arrowheads) and a left atrial tumour (asterisk). (B) Microscopically, small cell carcinoma forms intravascular thrombus in the distal part of the right pulmonary vein. (Stain: H\&E; magnification $\times 10$ ).

Lung cancer is one of the most common causes of metastatic cardiac tumours, but very few cases of intracardiac extension via the pulmonary vein are seen. If left untreated, patients may die from sudden cardiac arrest due to cardiac inflow obstruction or massive tumour emboli involving the major organs. ${ }^{12}$

\section{Learning points}

- Small cell lung cancer, though rare, can develop intravascular cancerous thrombus along the pulmonary vein with intracardiac extension.

- Timely surgical intervention can be life-saving to prevent patients from sudden cardiac arrest due to intracardiac extension of lung cancer.

\section{M-T Lin, ${ }^{1,3}$ S-C Ku, ${ }^{1}$ M-Z Wu, ${ }^{2}$ C-J Yu ${ }^{1}$}

${ }^{1}$ Division of Chest Medicine, Department of Internal Medicine, National Taiwan University Hospital, Taipei, Taiwan; ${ }^{2}$ Department of Pathology, National Taiwan University Hospital, Taipei, Taiwan; ${ }^{3}$ Department of Internal Medicine, Ear Eastern Memorial Hospital, Taipei County, Taiwan

Correspondence to: Dr S-C Ku, Division of Chest Medicine, Department of Internal Medicine, National Taiwan University Hospital, No 7 Chung-Shan South Road, Taipei 100, Taiwan; scku1015@ntu.edu.tw

Competing interests: None.

Patient consent: Obtained from patient's next of kin.

Accepted 19 November 2007

Thorax 2008;63:1122. doi:10.1136/thx.2007.090373

\section{REFERENCES}

1. Kodama K, Doi O, Tatsuta M. Unusual extension of lung cancer into the left atrium via the pulmonary vein. Int Surg 1990;75:22-6.

2. Brandt RR, Rubin J, Reeder GS. Intracardiac extension of a lung tumor causing left ventricular inflow obstruction. J Am Soc Echocardiogr 1995;8:930-3. 